\title{
UNO STUDIO SUL TERREMOTO DI CEFALONIA (DEL 12 AGOSTO 1953) CON PARTICOLARE RIGUARDO ALLA NATURA FISICA DELLA SCOSSA ALL'IPOCENTRO $\left({ }^{*}\right)$
}

\author{
D. Di Filippo - L. Marcelli
}

Introduzione - Macrosismica e isosiste. - La Grecia è una terra tristemente nota per la sua altissima sismicità e per i suoi frequenti e lunghi periodi sismici.

Tutti ricordano indubbiamente, tra gli ultimi, il periodo che ebbe inizio il 9 agosto 1953 e che scolvolse, per interminabili settimane, con convulsioni apocalittiche, la tormentata zona delle isole Jonie.

Sfogliando i giornali dell'epoca si ha una visione tragica dellimmane sciagura che per griorni e giorni, con ritmo inizialmente crescente, si è abbattuta sulle popolazioni indifese, rese folli dal terrore, schiantando case, polverizzando villaggi e città, spaccando montagne, divampando paurosi incendi, sollevando maremoti, travolgendo migliaia e migliaia di persone.

Argostoli, la capitale della Cefalonia, viene praticamente rasa al suolo, i villaggi di Agia ed Eftimia sono rimasti completamente distrutti; nell'isola di Zante altrettanto è accaduto per molti villaggi di pescatori situati sul braccio di mare che separa l'isola dalle coste del Peloponneso. Le popolazioni sono all'inizio prive di soccorso perché i crolli di grandi massi nei porti rendono pericoloso l'approdo delle prime barche accorse. Si attendono le unità della marina da guerra, e intanto i primi soccorsi vengono paracadutati dagli aerei dell'aviazione militare. S'improvvisano ospedali dove vengono assistiti centinaia, migliaia di feriti. Anche Lixouri e Samis sono distrutte. I villaggi dell'interno, per l'impraticabilità delle strade sconvolte, sono riforniti con elicotteri. L'isola di Itaca è gravemente danneggiata anche dal maremoto. Delle 2000 case di Vathy, la capitale, ne restano in piedi 50 : manca la luce, l'acqua, i viveri, i medicinali. La popolazione dell'isola, in preda al panico e alla disperazione, implora il trasferimento sulla terraferma. Zante, Cefalonia, Itaca sono ormai ridotte ad ammassi di

(*) Comunicazione presentata alla "Association Internationale de Séismologie et Phisique de l'intérieur de la Terre » nella X Assemblea Generale dell' U.G.G.I. • Roma 1954. 
macerie, divorate dalle fiamme di giganteschi incendi. Da Itaca moltissime persone si gettano in acqua nel disperato tentativo di raggiumgrere a nuoto le uniti di soceorso allancora fuori dei porti distrutti.

La gente di Cefalonia assiste ad un'altra terrificante visione: la montagna di Acnos, alta $1620 \mathrm{~m}$ si spacca. Altre montaqne di Cefalonia,

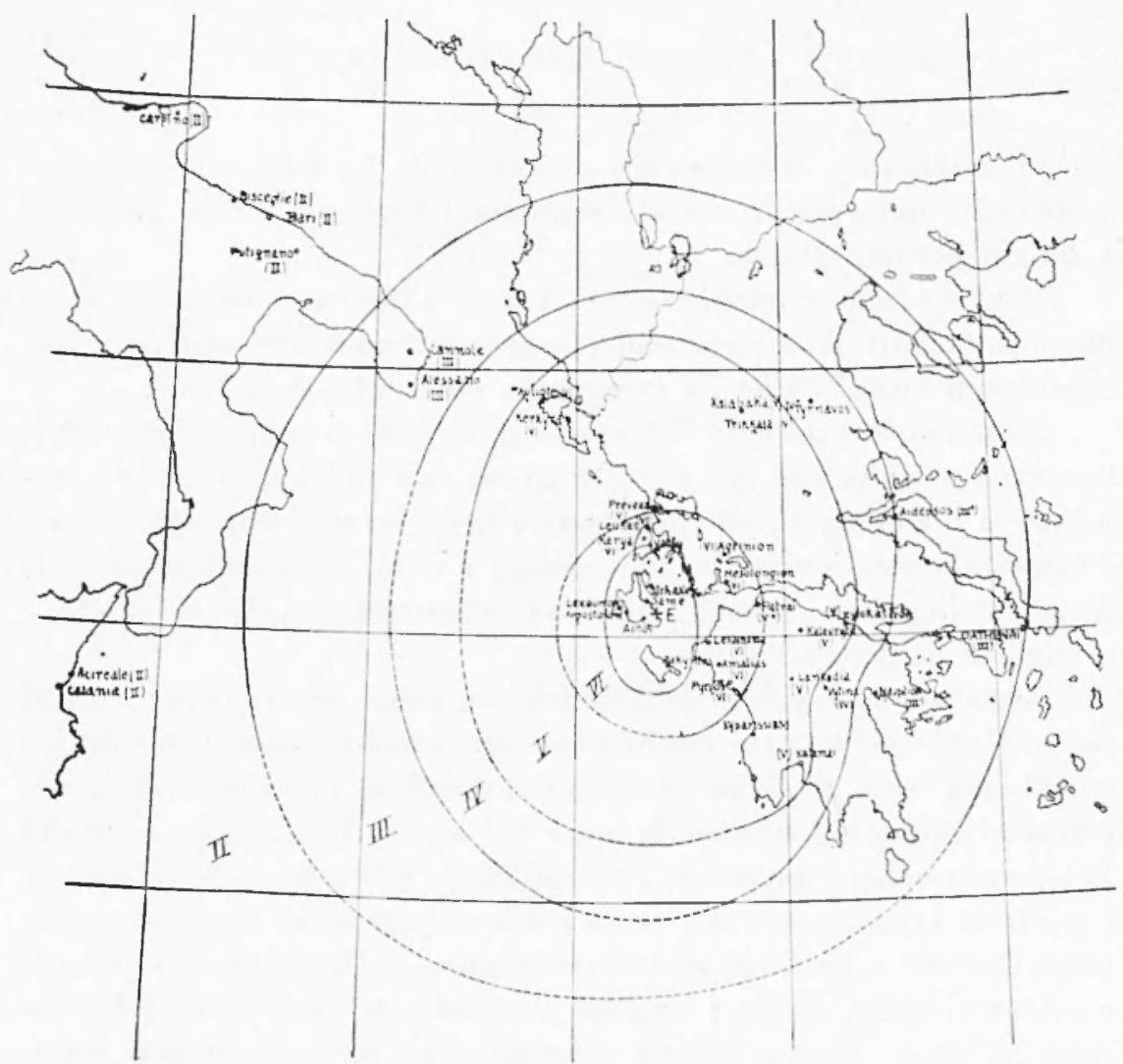

Fig. 1

Zante, Itaca franano o si aprono. "La distruzione è terrificante, totale, completan.

Linteresse che presenta la zona dal punto di vista scientifico ci ha indotto a polarizzare la nostra attenzione su qualcuna di queste scosse, si da farne oggretto di uno studio accurato e, per quanto possibile, esauriente.

A tale scopo, infatti, ci siamo procurate le registrazioni delle 3 scosse più forti, e precisamente quelle del 9 agosto $\left(H=07^{1 \mathrm{H}} 41^{\mathrm{m}} 06^{\circ} \mathrm{ca}\right.$ 
G. C. T.), dell'11 agosto ( $H=03^{\mathrm{h}} 32^{\mathrm{m}} 20^{\mathrm{s}}$ ca. G. C. T.) e del 12 agosto $\left(H=09^{\mathrm{h}} 23^{\mathrm{m}} 50^{\mathrm{s}}\right.$ ca. G. C. T.).

Fatto lo spoglio dei sismogrammi abliamo però deciso di tralasciare (almeno per ora) lo studio di quelli del 9 e dell'll agosto, perché gli inizi con i quali essi si presentano nei vari Osservatori non sono troppo chiari. Nettamente migliori - seppure non sempre bellissimi sono invece gli impulsi iniziali relativi alla scossa del 12 agosto, scossa di magnitudo più elevata delle precedenti.

È per questo precipuo motivo che il nostro studio si riferisce alla sola scossa del 12 agrosto.

In questo primo lavoro ci proponiamo di stabilire alcuni elementi essenziali che potranno servire, in seguito, per ulteriori indagini.

Da quanto $\grave{e}$ stato esposto in precedenza risulta chiara la impossibilitì di separare macrosismicamente una dall'altra, le centinaia di scosse che si sono susseguite quasi ininterrottamente per il lunghissimo periodo sismico iniziatosi la mattina del 9 agosto alle $07^{11} 41^{m} 05^{*}$ ca (G. C. T.) con una scossa di magnitudo $6-61 / 4$.

È evidente che le notizie macrosismiche riferentisi al terremoto più violento, sono anche la somma degri effetti precedenti. Solamente la magnitudo, calcolata sui sismogrammi per ogni scossa, può dare una indicazione analitica delle intensità relative ad ogni movimento.

Comunque, dai dati gentilmente fornitici dall'Osseratorio di Atene, ahliamo potuto tracciare il consueto quadro delle isosiste (v. fig. 1 ).

Il rạrgio di percettibilità è molto grande, essendosi la scossa sentita macrosismicamente perfino in alcune localita dell'Italia meridionale (di IJI in provincia di Fogrria e di Lecce, di II in provincia di Bari, Catania e Rag̨usa).

In numerosi osservatori, a distanze anche superiori ai $1000 \mathrm{Km}$, sono saltate le pennine dei registratori meccanici.

Calcolo dellipocentro e del tempo origine. - Per il calcolo delle coordinate epicentrali, della profondità ipocentrale e del tempo origine, ci siamo serviti del consueto metodo di Caloi-Peronaci $\left({ }^{1}\right.$ ) (usato ormai normalmente per $i$ rantaggi di calcolo che presenta sugli altri metodi probabilistici) facendo uso di coordinate geocentriche.

È noto che, scelto opportunamente un numero $n$ di stazioni sismiche, si deve giungrere a risolvere il seguente sistema di equazioni di condizione:

$$
\begin{gathered}
\delta t_{0}-\cos \varphi_{0}^{\prime} \sin \alpha \frac{\partial f}{\partial \Delta_{n}} \delta \lambda_{0}-\cos \alpha \frac{\partial f}{\partial \Delta_{n}} \delta \varphi_{0}^{\prime}+\frac{\partial f}{\partial h_{0}} \delta h_{0}= \\
=T_{n}-\left[t_{0}+f\left(\lambda_{0}, \varphi_{0}^{\prime}, h_{0}\right)\right]
\end{gathered}
$$


Nella [1] i simboli hanno i seguenti significati: $\delta t_{0}, \delta \lambda_{0}, \delta \varphi^{\prime}{ }_{0}, \delta h_{0}$ sono le correzioni da apportare rispettivamente al tempo origine $t_{0} e$ alle coordinate ipocentrali geocentriche $\lambda_{0}, \varphi^{\prime}, h_{0}$ scelte come valori iniziali :

$\sin x=\frac{c \cdot s \varphi \sin \left(\Lambda-\lambda_{0}\right)}{\sin \Delta}\left(\lambda\right.$ e $\rho^{\prime}$ essendo le coordinate geocentriche di ogni singola stazione, \& la distanza epicentrale geocentrica, $\propto$ l'azimut di ogni singola stazione rispetto all'epicentro).

$f\left(\lambda_{v}, \varphi^{\prime}{ }_{o}, h_{0}\right)$ è il tempo di propagazione delle $P n$ corrispondente alla $S_{v}$ (calcolata) della stazione considerata.

$\frac{\partial t}{\partial J_{\mathrm{a}}}$ è (per $h$ costante) l'incremento che subisce il tempo di propagazione (relativo ad ogni stazione) quando $\perp$ aumenta di 1 grado.

$\frac{\partial t}{\partial h_{\mathrm{o}}} \dot{\mathrm{e}}$ (per $\Delta$ costante) l'incremento che subisce ogni tempo di propagazione quando $h$ viene incrementata di una quantità pari al. l'unità della dromocrona prescelta.

$T n$ è il tempo di arrivo delle $P$ registrate dalle varie stazioni.

Le altre grandezze sono state rilevate dalle tavole.

Gli elementi ipocentrali dai quali siamo partiti per una prima approssimazione sono $i$ seguent $i$ :

$$
\left\{\begin{array}{l}
\varphi_{0}=38^{\circ}, 5 \mathrm{~N} \\
\lambda_{0}=21^{0} \mathrm{E} \\
t_{0}=09^{\mathrm{L}} 23^{\mathrm{m}} 50^{\mathrm{s}}: 5 \\
h_{0}=16 \mathrm{~km}
\end{array} \quad\right. \text { (in coordinate geografiche) }
$$

I primi due, $\varphi_{0}$ e $\lambda_{0}$, sono le coordinate fornite dall'U.S.C.G.S.

Il valore della profondita ipocentrale lo abliamo preso di $16 \mathrm{~km}$ considerando come dromocrona corretta quella di Jeffrey's ( $\left.{ }^{2}\right)$ relativa alla crosta terrestre, essendo evidente, dallo sviluppo delle registrazioni, trattarsi di terremoto superficiale.

Per quanto riguarda il valore del tempo origine, abbiamo ritenuto opportuno eseguire una media ponderale tra i vari tempi origine calcolati per ogni Osservatorio in quanto, le stazioni di cui ci siamo serviti, non sono distribuite regolarmente attorno all'epicentro, come risulta chiaramente dalla tabella $I$.

Delle 15 stazioni prescelte abbiamo calcolato le distanze epicen- 
trali goocentriche e gli azimut. Per i valori di $f\left(\lambda_{0}, \varphi^{\prime}{ }_{0}, h_{0}\right), \frac{\partial f}{\partial \dot{\Delta}_{n}}$, e $\frac{\partial f}{\partial h_{0}}$ ci siamo valsi delle dromocrone di Jeffrey's.

Una prima valutazione di $\delta h_{0}$ ci ha condotto ad un risultato negativo $\left(\delta h_{0}=-76 \mathrm{~km}\right)$ che ci autorizza a ritenere l'ipocentro in superficie, a profondita normale, mentre le coordinate epicentrali restano pressoché immutate; abbiamo dunque senz'altro rifat to i calcoli eliminando nella [1] il termine relativo a $\delta h_{1,}$.

Nella tabella I abbiamo riportato i soli dati indispensabili per giungere al sistema di equazioni di condizione.

TABELLA I

\begin{tabular}{|c|c|c|c|c|}
\hline Stazioni & $\begin{array}{c}\lrcorner \\
\text { geocentrica } \\
\text { provvisoria }\end{array}$ & $\alpha$ & $\begin{array}{c}\text { tempo di } \\
\text { arrivo della Pn } \\
\text { sul sismo- } \\
\text { gramma }\end{array}$ & errori \\
\hline 1) Messina & $40,29+4$ & $2670+9^{\prime} 00^{\prime \prime}$ & $09 \mathrm{~b} 2+\times 155^{\sharp}, 5$ & $-0,36129$ \\
\hline 2) Triestc & 8,9383 & 3251610 & 2604,5 & $+0,86750$ \\
\hline 3) Zurigo & 12,6950 & $324+853$ & 2656 & $\div 0,82741$ \\
\hline 4) Praga & $12,+805$ & $3+03+4$ & 2655,5 & $-0,31537$ \\
\hline 5) Stoccarda & $13,3+44$ & $32+06+9$ & $270+, 5$ & $\div 0,88015$ \\
\hline 6) Algeri & 14,3344 & 26729 & $27 \quad 12,9$ & $+0,66960$ \\
\hline 7) Srasburgo & 13,9011 & $320+505$ & 2713,4 & $-0,96824$ \\
\hline 8) Gottinga & 15,1769 & 3325030 & 2729,2 & $\div 0,90997$ \\
\hline 9) Kew Observ. & 19,7917 & $317+823$ & $28 \quad 26,9$ & $-0,87410$ \\
\hline 10) Tamanrasset & 20,5092 & $224+106$ & 2829,4 & $-0,76074$ \\
\hline 11) Uppsala & $21,+850$ & 3552127 & $28+5,6$ & $+0,145+7$ \\
\hline 12) Almeria & $18,6+53$ & 26732 & $\begin{array}{ll}28 & 07,7\end{array}$ & $+0,91992$ \\
\hline 13) Tananarive & 62,3039 & 1512716 & $34 \quad 15$ & $+0,46689$ \\
\hline 14) Rcykjavik & 35,9822 & 3292729 & $30 \quad 59,2$ & $-2,96675$ \\
\hline 15) New Delhi & 47,2333 & $8+3520$ & $32 \quad 28,3$ & $+0,55952$ \\
\hline
\end{tabular}

Risolto con il solito metodo dei minimi quadrati, e calcolati successivamente gli errori probabili per ciascuna delle incognite, si ottiene:

$$
\begin{aligned}
& \delta \varphi_{0}^{\prime}=-0^{\prime \prime}, 32529 \pm 0^{\prime \prime}, 04529 \\
& \delta \lambda_{0}=-0^{\prime \prime}, 27985+0^{\prime \prime}, 06738 \\
& \delta t_{\mathrm{o}}=-0^{\circ}, 14968 \pm 0^{\circ}, 63983
\end{aligned}
$$


con [11. 3] $=16,1987 \mathrm{e}[\mathrm{w} \mid=16,1986$ come è richiesto dalla teoria degli errori.

Arremo quindi, per l'ipocentro, i seguenti valori definitivi:

Coordinate geografiche

$\varphi_{0}=+38^{\prime} 10^{\prime} 26^{\prime \prime}, 8+2^{\prime} 43^{\prime \prime}, 04$

$\lambda_{\mathrm{o}}=+20^{\prime \prime} 43^{\prime} 12^{\prime \prime}, 54+4^{\prime} 02^{\prime \prime}, 57$

$h_{\mathrm{o}}=0$

$t_{\mathrm{o}}=09^{\mathrm{h}} 23^{\mathrm{m}} 50^{\mathrm{s}}, 35032 \div 0^{\mathrm{s}}, 63983$
Coordinate geocentriche

$$
\varphi_{0}^{\prime}=37^{\prime \prime} 59^{\prime} 10^{\prime \prime}, 3+2^{\prime} 43^{\prime \prime}, 04
$$$$
\lambda_{\mathrm{o}}=20^{\prime \prime} 43^{\prime} 12^{\prime \prime}, 54+4^{\prime} 02^{\prime \prime}, 57
$$

$h_{\mathrm{o}}=0$

$t_{0}=09^{\mathrm{n}} 23^{\mathrm{m}} 50^{*}, 35 \div 0^{*}, 64$

Dall'ultime colonna della tabella I risalta la buona approssimazione dei risultati conseguiti. Gli errori, ad eccezione della stazione di Reykjavik, sono tutti inferiori all’unità, con ragrionevole ripartizione di valori positivi e valori negativi.

Energia erogata durante tutto il periodo sismico agosto-settembre. La magnitudo di questo terremoto, calcolata in 8 diverse stazioni della rete sismica internazionale ha un valore medio di

$$
7.24 \sim 71 / 4
$$

pari ad una energria erogata di circa

$$
10^{-\cdot-\cdot} \text { erẹ. }
$$

Abbiamo voluto fare un computo approssimato dell'energia to* tale sviluppata per tutto il periodo sismico che va fino alla fine di settembre.

In questo calcolo abbiamo tenuto conto anche delle scosse di piccola intensità.

Ci siamo valsi della documentazione fornita dal Bollettino di Atene e da quello dell'I.N.G. ritenendo che, data la vicinanza dell'Osservatorio di Messina alla zona epicentrale, e data l'ottima efficienza strumentale di detta stazione, ben poche scosse possano essere sfuggite alla catalogazione. E comunque quelle eventuali scosse tralasciate, essendo 
per ovvie ragioni di modestissima magnitudo, portano un contributo addirittura insignificante al computo globale dell'energia.

Ricordando la suddivisione in classi che Gutenberg $\left({ }^{3}\right)$ propone per i terremoti a seconda della loro magnitudo (*) si può fare una prima catalogazione delle scosse relative al periodo sismico in questione.

La scossa principale appartiene alla classe $b$ ed è l'unica: vi sono poi 4. scosse di classe $c, 54$ di classe $d, 316$ circa di classe $e$.

Più dettagliatamente l'analisi porta:

\begin{tabular}{|c|c|c|c|c|c|c|c|c|c|c|c|c|c|}
\hline 1 & scossa & di & magnitudo & 7,24 & $\operatorname{con} c$ & erogazione & $\mathrm{di}$ & una & energia & pari & a & 1024,7 er & rg. \\
\hline 1 & " & " & , & 6,79 & " & " & " & $"$ & , & 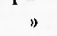 & s & 1023,8 & 9 \\
\hline 1 & $"$ & $"$ & 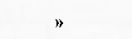 & 6,36 & $"$ & $\Rightarrow$ & " & " & $"$ & 3 & " & 1022,9 & $"$ \\
\hline 1 & $"$ & " & " & 6,34 & $"$ & $"$ & $n$ & $"$ & $"$ & $"$ & $"$ & 1022,85 & $"$ \\
\hline 1 & $"$ & , & s & 6,1 & $"$ & $"$ & $"$ & $"$ & $"$ & - & 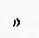 & 1022,35 & $"$ \\
\hline 1 & $"$ & $n$ & , & 5,78 & " & $"$ & $\Rightarrow$ & , & $"$ & $"$ & $"$ & 1021,70 & $"$ \\
\hline 1 & ") & $"$ & $\Rightarrow$ & 5,75 & , & $"$ & " & " & $"$ & " & $\Rightarrow$ & 1021,68 & $"$ \\
\hline 2 & $"$ & " & , & 5,62 & , & $"$ & $"$ & $"$ & $"$ & $"$ & $"$ & $2 \times 1021,4$ & $"$ \\
\hline 1 & $"$ & " & $"$ & 5,60 &, & $"$ & $\Rightarrow$ & $"$ & $"$ & $"$ & $"$ & $10 \geq 1,4$ & $"$ \\
\hline 1 & " & " & " & 5,56 & $"$ & $"$ & $"$ & " & $"$ & " & " & 1021,3 & " \\
\hline 17 & $n$ & $"$ & $"$ & 5.511 & $b$ & $"$ & , & ") & $"$ & $"$ & " & $17 \times 1021,2$ & " \\
\hline 2 & , & $\Rightarrow$ & s & 5,38 & , & $"$ & $"$ & $"$ & " & $"$ & $"$ & $2 \times 1021$ & $"$ \\
\hline 29 & 1) & $"$ & $"$ & 5,30 & $"$ & $"$ & $"$ & $"$ & $"$ & $"$ & " & $29 \times 1020,8$ & $"$ \\
\hline 15 & " & $"$ & $"$ & $5,(11)$ & $"$ & , & $"$ & $"$ & " & $"$ & , & $15 \times 1020,2$ & . \\
\hline 21 & $"$ & $"$ & " & 4.511 & $"$ & " & " & $"$ & " & $"$ & $"$ & $21 \times 1019,2$ & $n$ \\
\hline 33 & $"$ & $"$ & " & $4,(1)$ & $"$ & , & $"$ & $"$ & $\Rightarrow$ & " & " & $33 \times 10^{1 \mathrm{~S}, 25}$ & $"$ \\
\hline 48 & $n$ & $"$ & $"$ & 3.50 & $"$ & ") & $:$ & $"$ & $"$ & $"$ & s & $48 \times 10^{17,3}$ & $"$ \\
\hline 49 & $"$ & $"$ & , & 3,000 & " & $\Rightarrow$ & $\Rightarrow$ & $"$ & $\Rightarrow$ & " & $n$ & $49 \therefore 10^{16,3}$ & $n$ \\
\hline $150 \mathrm{ca}$. & $1)$ & $"$ & , & $2,51)$ & $\Rightarrow$ & , & ; & $"$ & $\therefore$ & , & $"$ & $150 \times 1015,3$ & $"$ \\
\hline
\end{tabular}

Natura della scossa allipocentro. - Un esame molto accurato degli impulsi iniziali sui sismogrammi delle varie stazioni, è stato eseguito allo scopo di individuare la natura della scossa allipocentro. In effetti moltissime stazioni, pur avendo delle ottime rewistrazioni, presentano un inizio non eccessivamente chiaro. Negli osservatori più ricini e meglio dotati infatti, il grafico si presenta con un legrgerissimo impulso iniziale, che spesso è addirittura una "emersio " seguita subito dopo da un nettissimo "impetus", molto ampio. Nella fig. 2 vengono riportate come esempi le registrazioni delle stazioni di Chur, Strasbourg. Questo fatto naturalmente comporta che là dove wli scorrimenti non sono sufficientemente grandi o la sensibilità dello strumento è scarsa, l'inizio è stato addirittura assorbito e ciò che può rite-

(*) Nella classe a vinno posti i terremoti di magnitudo compresa tra $71 / 2$ e $8 \frac{1}{2}$; nella classe $b$ quelli tra 7,0 e 7,7 ; nella classe $c$ quelli tra 6,0 e 6,9 ; nella classe $d$ quelli tra 5,3 e 5,9 ; nella classe $e$ quelli minori di 5,3 . I terremoti di classe $e$ non sono registrati oltre i $10^{\circ}$. 
nersi l'inizio non è che il secondo impeto. Per evitare ambiquità di interpretazioni abbiamo quindi proceduto ad una accuratissima indagine, aiutandoci, dore l'incertezza sussisteva, con il calcolo dei tempi d'arrivo in base al tempo origine trovato.

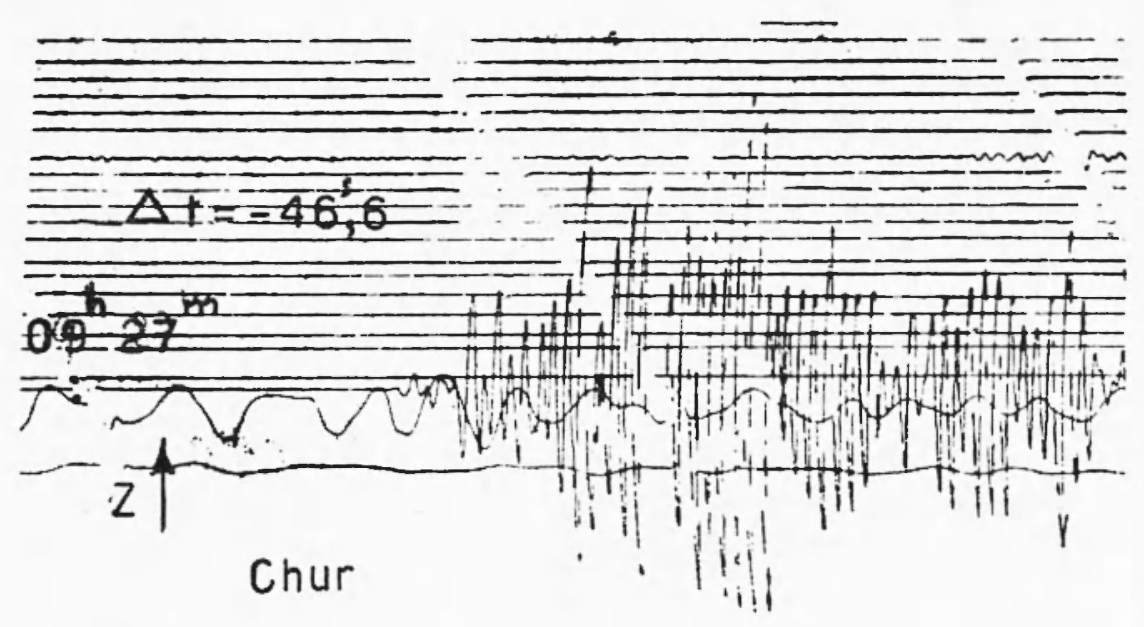

$\Delta t=+1.6$

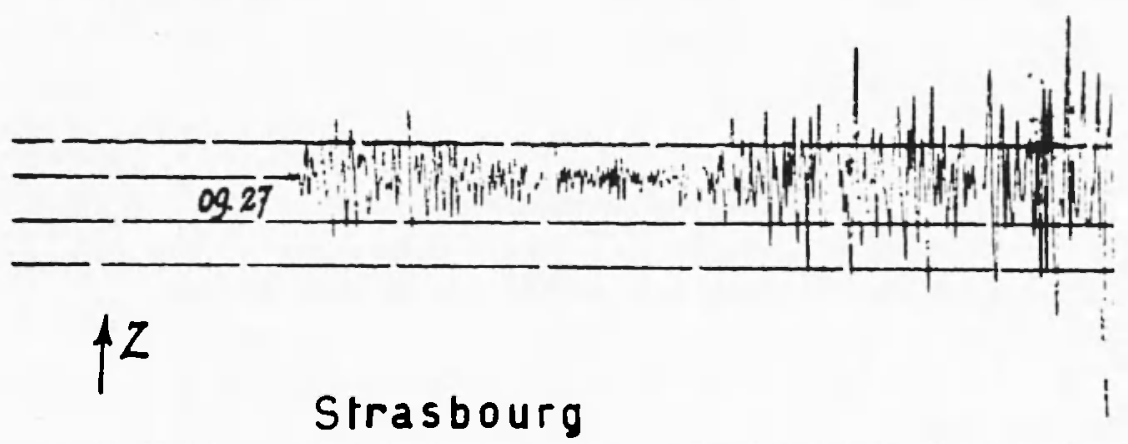

Fig. 2

Abbiamo potuto quindi disegnare, con una discreta precisione, la fig. 3 dalla quale risulta chiaramente una distribuzione abliastanza regolare di dilatazioni intorno alla zona epicentrale, e, a distanza, tutte compressioni.

Sono infatti nette dilatazioni: Atene (1), Regroio Calabria (2), Messina (3), Roma (4), Firenze (5), Pavia (6), Trieste (7), Vienna (8), Helwan (9), Algeri (10), Almeria (11), Lisbona (12), Tortosa (13). 
Compressioni nei seguenti osservatori: Chur (14), Neuchâtel (15), Zurigo (16), Stoccarda (17), Strasburgo (18), Parigi (19), Lccle (20), Gottiga (21), Jena (22), Praga (23), Kew (24), Dublino (25), Kopenhagen (26), Uppsala (27), Reykjavik (28), Tamanrasset (29), New Delhi (30), Fukuoka, Kodaicanal, Shasta, Ottawa, Riverwiev, Palisades.

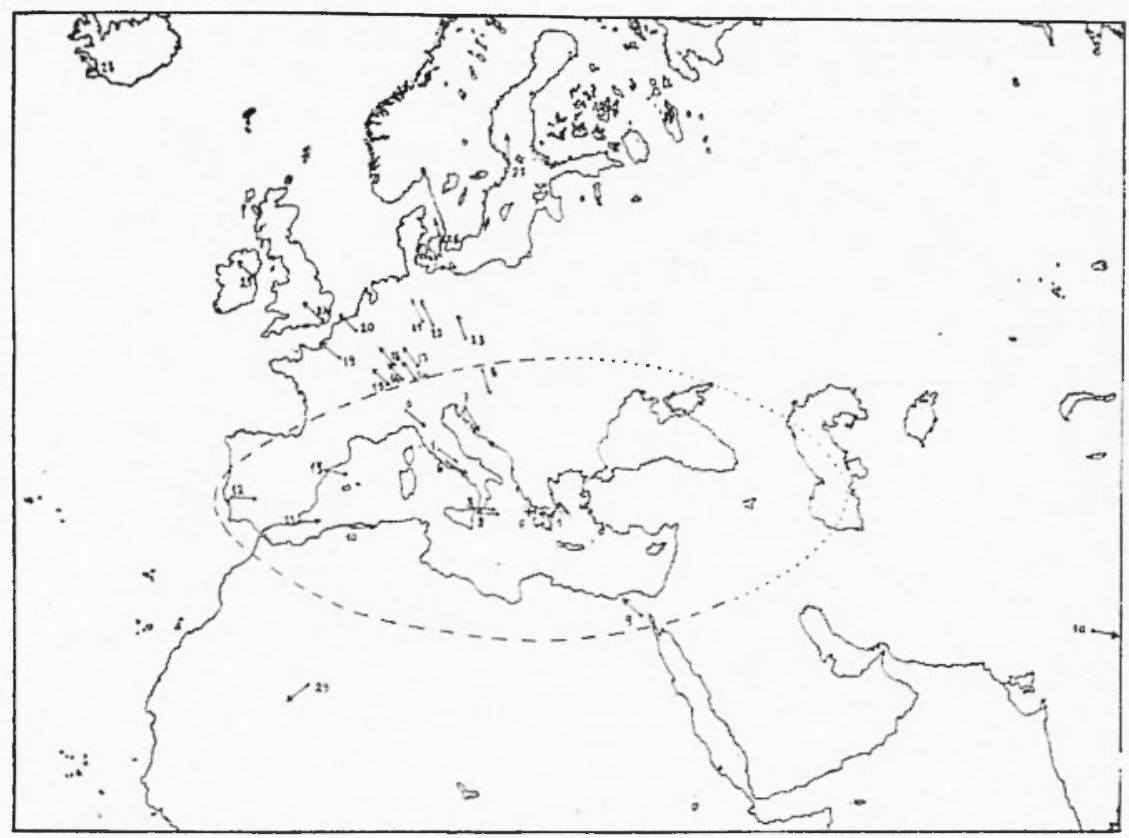

Fig. 3

Questa distribuzione è caratteristica dei moti di sprofondamento, sicché siamo portati a supporre, con sufficiente attendibilità, che l'origine della scossa si possa attribuire ad un vero e proprio sprofondamento.

E interessante inquadrare questo risultato tra quelli conseguiti nei vari studi sui terremoti dell'Europa Centro-Meridionale e dell'Italia. Nella riunione tenuta a Stoccarda nel settembre del 1952, indetta dalla Commissione Sismolorica Europea, Caloi ha presentato una relazione $\left({ }^{*}\right)$ in cui sintetizza $i$ risultati delle ricerche effettuate da studiosi italiani sul problema delle caratteristiche geologico-sismiche del continente europeo, con particolare riguardo per l'Italia. 
La fig. 4 è uguale a quella da lui presentata ma completata dal nostro risultato.

È evidente un allineamento di sprofondamenti che sembra seguire la direzione del mare Adriatico, mentre i terremoti con epicentro sugli Appennini si presentano con sollevamenti, lungo una fascia pressoché parallela. Caloi nella sua citata esposizione fa notare una pro-

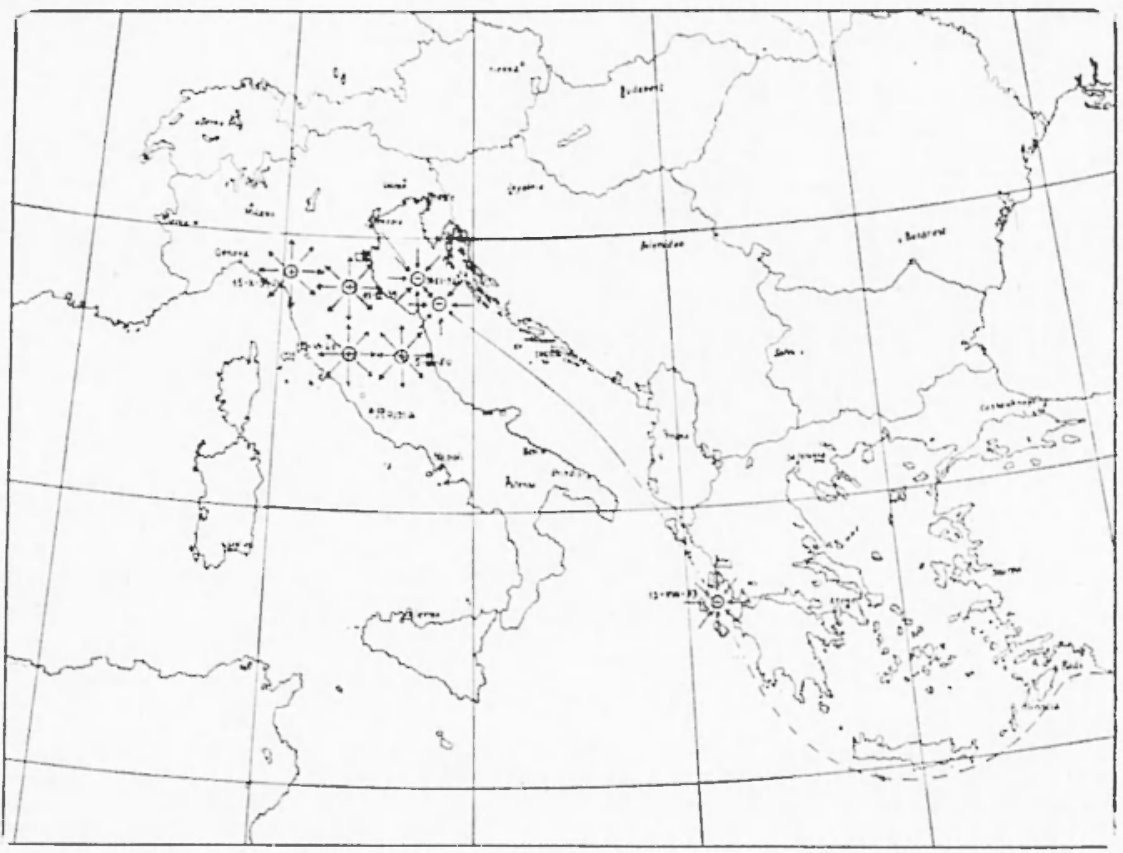

Fig. 4

babile relazione tra gli sprofondamenti dell'alto Adriatico e i sollevamenti della catena Appenninica. "Sembra quasi che alle compressioni laterali determinate dai primi conseguano i sollevamenti causati dai secondi. A meno che le qualifiche di causa ed effetto non delbbano essere invertite ).

Noi, da parte nostra, ci limitiamo ad aggiungere agli altri il nustro risultato. Mancano studi di tal genere eseguiti su terremoti con epicentro nel basso Appennino per poter asserire la continuiti delle mutue relazioni su esposte.

Certo lo sprofondamento del terremoto da noi ora studiato è allineato con quelli dell'alto Adriatico, ma si può associare a quelli nel vasto fenomeno di orogenesi a cui indubbiamente partecipa la peni- 
sola italica? oppure lo si deve inquadrare nella storia geologica dell'Egeide?

E altresi probabile che l'una ipotesi non escluda l'altra.

Considerazioni geologiche. - Pù̀ essere molto interessante esporre qualche rilievo geologico che potrebbe dar credito al nostro risultato.

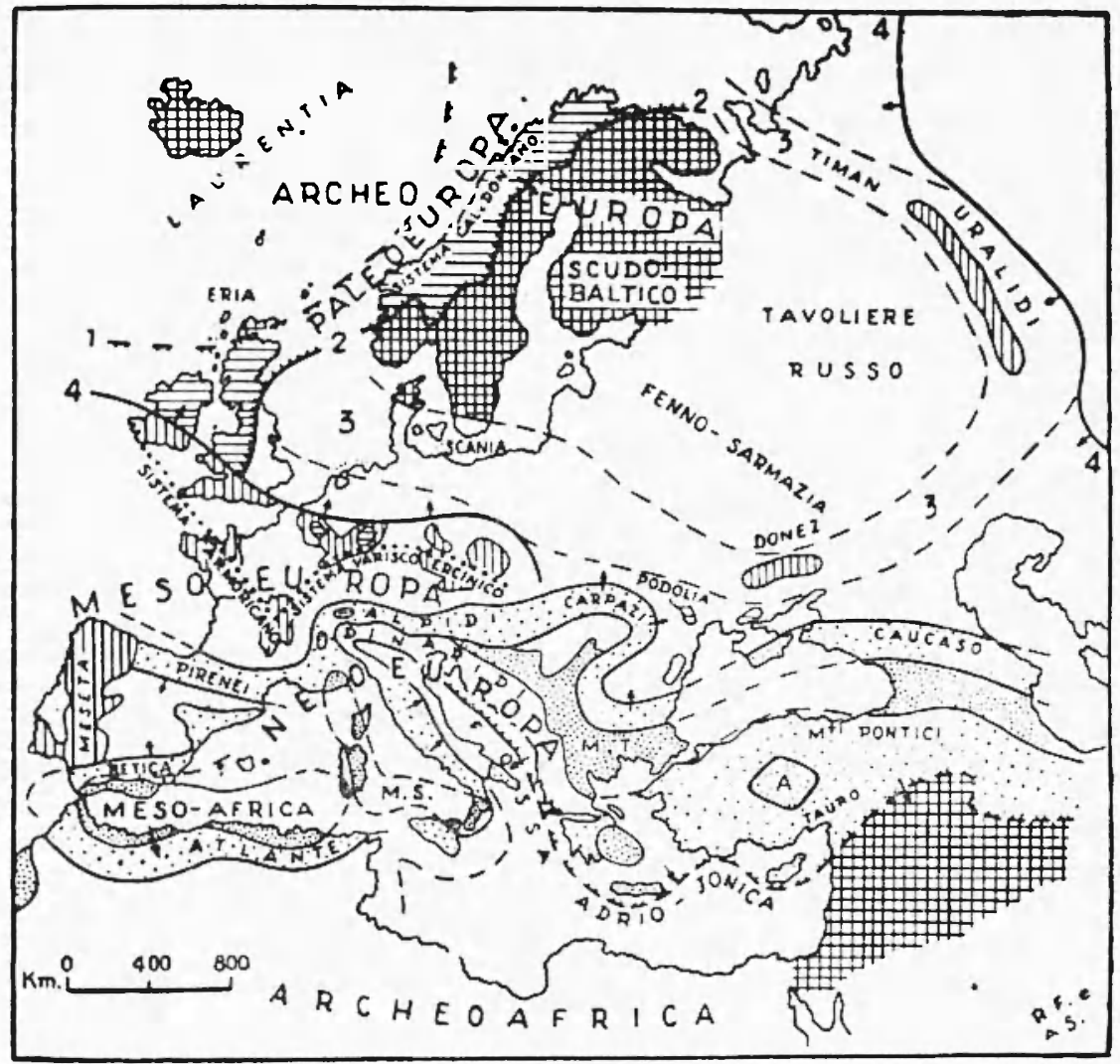

Fig. 5

Degna indubbiamente di rilievo, ai nostri fini, è la carta geologica europea che riportiamo nella fig. 5 e che trovasi pubblicata sul " Trattato di geologia " di Ramiro Fabiani. È ivi messa in luce una linea geosinclinale indicante la fossa Adrio-Jonica la quale, controllata fino all'altezza delle Puglie, subisce poi un presupposto piegamento lungo i contorni della Grecia continentale, del Peloponneso, di Creta: lam- 
bisce Rodi, le coste meridionali di Cipro e si insinua poi nel continente a delimitare inferiormente i corrugamenti terziari della Turchia e del Caucaso.

Notiamo come gli sprofondamenti si allineino esattamente su tale sinclinale, talché ci sembra evidente che essa si possa considerare controllata almeno fino alla zona epicentrale del terremoto in studio.

Purtroppo non molto abbondante è la letteratura geologica sulla regione che a noi interessa. Molti grli studiosi che hanno rivolto la loro attenzione sulla storia geologica dell'Egeide, ma mancano studi particolari sulla parte occidentale della regione.

$\mathrm{Ci}$ è sembrata di un certo interesse anche una nota del 1930 che il geologo italiano Carlo Mirrliorini (") fece sull'isola di Alinnià (il più grande degrli isolotli disseminati tra Rodi e Calchil. Egli asserisce che le caratteristiche dell'isola sono tali da dimostrare come l'isola stessa abbia subito recentemente un abbassamento complessivo. Errli dice iestualmente:

"Ritengro che l'abbassamento sia dovuto allo sprofondamento a zolle al quale andò soggetta, nel post-pliocene, la regione egea ". Alinnià sarebbe quindi un'isola di smembramento tettonico.

Altri interessanti rilievi furono eseguiti in occasione del $46^{\circ}$ Congresso della Società Geologica Italiana tenutosi a Rodi sotto la presidenza dell'illustre geologo Alessandro Martelli ( ${ }^{\circ}$ ).

La costa albanese, intorno a Santi Quaranta, presenta delle analogie morfologiche e geologiche con il Carso costiero di Trieste. La roccia che compone il territorio costiero è di natura calcarea relativa al periodo Cretaceo superiore ed Eocenico inferiore e medio. Verso il canale di Corfù compaiono lungo la costa terreni piu antichi.

Lungo i golfi di Patrasso e di Corinto che tagliano il Peloponneso dalla Grecia continentale, si notano interessanti diversità di aspetto tra le due sponde, diversità che secondo alcuni geologi andrebbero messe in relazione con un grande sollevamento del margine settentrionale del Peloponneso avenuto verso la fine del Pliocene: nella regrione costiera meridionale invece alcuni piani di faglia stanno ad indicare piani lungo $i$ quali si son venute sprofondando le zolle marginali del Peloponneso.

Il nostro epicentro si trova proprio ai margini occidentali del taglio tra Peloponneso e Grecia meridionale. Non potremmo dunque trovarci di fronte ad uno di questi fenomeni di sprofondamento? Certo, si tratta di supporre un perdurare dei movimenti di ororenesi, ma 
l'intensa sismicità attuale depone a favore di tale supposizione, né i geologi sono contrari ad ammetterlo.

Un accuratissimo studio geologico sulle isole di Rodi e di Coo effettuato in occasione del citato congresso li porta infatti a dire: "Una stretta relazione risulta tra le forme tettoniche neogenico-neozoiche (di Rodi) e la tettonica pre-neogenica (alpina). Relazione che conferma che le strutture tettoniche tardive di Rodi sono da considerarsi come manifestazioni di un vero periodo di orogenesi attenuata susseguente e geneticamente legato al periodo orogenico alpino... F da ritenere inoltre che l'intensa sismicità attuale sia un indizio del perdurare dei movimenti di orogenesi attenuata ).

Questi studi esaurienti purtroppo si riferiscono ad isole piuttosto lontane dalla zona delle isole Ionie, ma è lecito incuadrare le une e le altre nella storia geologica del Mediterraneo e dell'Egeide.

Alcuni concetti emessi dal grande geologo Suess sono oggi universalmente accettati.

Un unico mare, il cosidetto mar Tetys, si sarebbe esteso, nell'éra mesozoica, dall'America Centrale, e attraverso l'Europa fino al Golfo del Bengala. Nel periodo post-eocenico hanno avuto luogo i sollevamenti che hanno fatto parzialmente emergere le catene montuose del bacino Mediterraneo facenti parte di quei grandiosi gruppi di pieghe che dall'Atlante si congiungono all'Hymalaia lungo i Pirenei, le Alpi. i Carpazi, e il Caucaso tutti prevalentemente costituiti dai sedimenti di quel mare mesozoico il quale si sarebhe cosi ristretto fino a presentare i suoi residui attuali nel Mediterraneo, nel Mar Nero e nei maggiori laghi dell'Asia.

Sembra ormai incontestabile che al sorgere dell'éra quaternaria, a causa della persistenza delle spinte orogeniche che avrebbero intensificato la fagliatura, parziali affondamenti nell'area emersa abbiano condotto al frazionamento dell'Egeide (pare così che anche Rodi e altre isole dell’Egeo facessero parte di una stessa terrafermal.

Posteriormente a questo frazionamento, sarebbe ricominciato il sollevamento finché, col prevalere dell'erosione marina, i contorni delle isole si ridussero ulteriormente. Dopo l'epoca glaciale poi, l'intrusione del Mediterraneo nel bacino pontico avrebbe determinato lo stato di cose pressoché attuale.

È assolutamente accertato che il fenomeno della formazione dell'Egeide ebbe luogo verso la fine del terziario e all'inizio del quaternario, in epoca in cui già esisteva l'uomo.

Non tutti i geologi concordano però con Suess su la sua teoria 
degli sprofondamenti secondo la quale $i$ forti abhassamenti di estese masse lungo linee e piani di frattura sarebbero il precipuo fattore dei cambiamenti della superficie terrestre e di fronte ad esso la formazione di pieghe e il sollevamento delle montagne per contrazione laterale non sarebbe che un fenomeno secondario.

Infatti secondo altri autori (come il De Stefani e il Martelli) le terre dell'Egeo non si trovano isolate per fratture e per affondamenti, ma a causa di sollevamenti talché l'Egeo (che sarebbe sempre stato occupato da isole) tendereblue sempre più a restringersi per il prevalere del sollevamento della regione.

Da queste teorie, tutte ragionevoli dal punto di vista scientifico, risulta però che non ci sono dati così completi e sicuri da conscntire una esauriente storia geologica dell'Egreo.

Siamo lieti di apportare il nostro modesto contributo ad un problema cosi interessante.

Roma - Istituto Nazionale di Geofisica - Luglio 1954

\section{RIASSUNTO}

Allo scopo di iniziare uno studio sulla struttura interna della crosta terrestre in corrispondenza della zona ionica adiacente alla Grecia, è stato calcolato l'epicentro della scossa sismica che risultò, tra tutte quelle che nell'agosto 1953 colpirono le isole Jonie, quella di massima intensità.

Stabilito inoltre che il movimento in questione fu dovuto a sprofondamento, si raffronta questo risultato con quelli conseguiti studiando $i$ terremoti dell Alto Adriatico edella catena Appenninica. Completando lo studio con delle notizie geologiche, si nota come tutti gli sprofondamenti dell'Adriatico, collegati probabilmente con $i$ sollovamenti appenninici, si allineino lungo la geosinclinale costituente la fossu Adrio-Jonica. Non si esclude inoltre la possibilità che il fenomeno sismico si possa inqundrare nella storia geologica dell Egeide.

\section{SUMMARY}

For the purpose of initiating a study on the internal structure of the terrestrial crust which corresponds to the ionic zone adjacent to Greece, the epicenter of the sismic shock having the maximum 
intensity among all those which affected the island of Jonie in August 1953, has been calculated.

Ascertained further that the movement in question was due to sinking, this result was compared with those subsequently carried out studying the earthquahes of the Upper Adriatic and the Appenine chain. Completing the study with some geological facts, one notes how all the sinkings of the Adriatic, connected probably with the Appenine upheavals, are aligned along the geosyncline formed by the Adrio-Jonica fosse.

Further, the possibility that this sismic phenomenon can be included in the geologic history of the Aegean seas, cannot be excluded.

\section{BIBLIOGRAFIA}

(1) P. Chlor-F. Peroxici, Il terremoto del Turkestan del 2 notembre 1946. Annali di Geofisiea I, 2, 246 (1948).

(2) British Association for the Advancement of Science: Gray Milne Trust. H. JFFlpers and K. E. Butex, Seismological Tables. London 1940.

(3) B. Gutuxbsho, C. F. Richten, Seismicily of the Earht.

(1) P. CaLoI, Strutura geologico-sismica dellEuropa centro-meridionale, dell'talia e del Mediterraneo centro-occidentale, quale risulta da recenti studi di ricercatori italimi. Bull. d'Information de l'L.G.G.J., II Annee, n. 2, avril 1953.

(ii) C. MugLonns. Appunti geologici sullisola di Alinnia (Egeo) ed alcune considernzioni sulla geologin di Rodi. Boll. della Societa Geologica I..l., vol. XLIX, 1930, fasc. I.

(i) 1 XlIl Congresso della Soc. Geolog. Ltaliann. Boll. della Soc. Geol. Ital., Vol. I.11, 1933. 